\title{
Variational study of a two-level system coupled to a harmonic oscillator in a ultra-strong coupling regime
}

\author{
Myung-Joong Hwang ${ }^{1}$ and Mahn-Soo Choi ${ }^{2, *}$ \\ ${ }^{1}$ Department of Physics, Pohang University of Science and Technology, Pohang 790-784, Korea \\ ${ }^{2}$ Department of Physics, Korea University, Seoul 136-713, Korea
}

\begin{abstract}
The non-classical behaviors of a two-level system coupled to a harmonic oscillator are investigated in the ultra-strong coupling regime. We revisit the variational solution of the ground state and find that the existing solutions do not account accurately for non-classical effects such as squeezing. We suggest a new trial wave function and demonstrate that it has an excellent accuracy on the quantum correlation effects as well as on energy.

PACS numbers: 03.67.Lx, 42.50.Pq, 42.50.Dv, 85.25.Cp
\end{abstract}

A two-level system interacting with a harmonic oscillator appears in various fields in physics, ranging from an atom coupled to a photon inside of an optical cavity [1] to a cooper-pair box coupled to a nanomechanical oscillator [2]. A theoretical model that describes the system is expressed by the Hamiltonian,

$$
H=\omega_{0} a^{\dagger} a+\frac{1}{2} \Omega \sigma_{z}-\lambda\left(a+a^{\dagger}\right) \sigma_{x}+\lambda^{2}
$$

where $\omega_{0}$ is the energy of the oscillator, $\Omega$ is the spin level splitting, and $\lambda$ is the coupling strength. The Pauli matrices characterize the two level system, while $a$ and $a^{\dagger}$ denote the boson operators.

The Hamiltonian (1) reveals completely different physics at different scales of $\omega_{0}, \Omega$ and $\lambda$. For example, a cavity quantum electrodynamics (QED) operates in a regime where $\lambda,\left|\omega_{0}-\Omega\right| \ll \omega_{0}, \Omega$, under which the counter-rotating terms of the Hamiltonian $11, a^{\dagger} \sigma^{+}$and $a \sigma^{-}$, can be neglected. Within this rotating wave approximation (RWA), the model reduces to a Jaynes-Cummings model and is solvable exactly [3]. The ground state is a simple direct product of the ground states of the oscillator and the spin. On the other hand, in the so-called ultra-strong coupling regime $\left(\Omega \gg \omega_{0}\right.$ and $\lambda \sim \sqrt{\Omega \omega_{0}}$ ), there arise interesting quantum effects in the ground state; the two-level system and the oscillator are entangled. The degree of entanglement increases monotonically as a function of the dimensionless coupling constant $g \equiv 2 \lambda / \sqrt{\Omega \omega_{0}}$. It also shows a squeezing effect [4], that is, the variance of momentum quadrature in the ground state becomes smaller than the uncertainty minimum [5-77]. Hereafter, we will set $\omega_{0}=1$ for simplicity.

The aforementioned ultra-strong regime has recently attracted much attention because of the possibility to realize it experimentally in a circuit QED system [7-10]. Furthermore, as rather a theoretical problem, the ultra-strong coupling regime when $\Omega$ becomes $\infty$ has been also studied extensively because it shows a phase transition-like behavior in the entanglement between the oscillator and the qubit in the ground state [11--16].

\footnotetext{
*Electronic address: choims@korea.ac.kr
}

In this paper, we develop a variational ground state. The variational method had already been used for the Hamiltonian (11). A displaced coherent state for $g \gg 1$, a displaced squeezed state for $g \ll 1$ [17], and a superposition of two coherent states for $g \simeq 1$ [18, 19] have been suggested as a variational ground state, respectively. The accuracy of the suggested variational states were reasonable on the groundstate energy [17-19]. However, we find that the variational states suggested in the previous works [18, 19] underestimates the squeezing effect significantly in the intermediate regime ( $g \simeq 1$ ); see Figs. 2 (b) and (e). The failure gets more pronounced as $\Omega$ increases.

This observation implies that an energy-optimized variational wave function does not necessarily capture all the quantum correlation effects in the true ground state of the system. In this paper, we suggest a new variational ground state which captures accurately the squeezing effect.

First of all, we derive an equivalent model with only a boson degrees of freedom, followed by a derivation of an effective classical Hamiltonian by utilizing a parity symmetry in the model [20]. We observe that this effective classical Hamiltonian shows bifurcation around $g=1$, which is consistent with the observation in Ref. ([12, 14]) based on a classical model of the Eq. (1), and gives us qualitative account for the properties of the ground state. We then suggest a superposition of two displaced-squeezed state as a trial wave function, and demonstrate that it can predict the squeezing effect accurately. This shows that a deformation of each superposed wave packet is crucial to the squeezing effect of ground state. We also discuss the drastic change in the entanglement degree as $g$ varies across $g=1$ when $\Omega \rightarrow \infty$.

\section{EFFECTIVE HAMILTONIAN}

The model (1) has a useful symmetry. If $\left|n^{\prime}, \sigma^{\prime}\right\rangle=$ $H|n, \sigma\rangle$, then $n^{\prime}+\sigma^{\prime}(\bmod 2)=n+\sigma(\bmod 2)$, where $|n, \sigma\rangle \equiv|n\rangle \otimes|\sigma\rangle,|n\rangle(n=0,1,2, \cdots)$ is the boson number state, $a^{\dagger} a|n\rangle=n|n\rangle$, and $|\sigma\rangle(\sigma= \pm 1)$ the spin state, $\sigma_{z}|\sigma\rangle=\sigma|\sigma\rangle$. We further stress this symmetry by introducing a generalized "parity" operator

$$
\Pi=\exp \left(-i \pi a^{\dagger} a\right) \sigma_{z} .
$$



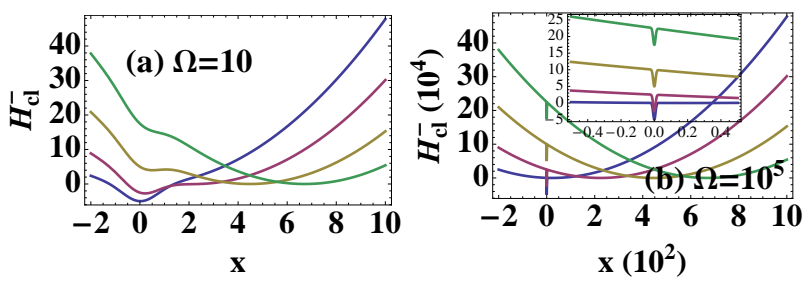

FIG. 1: (color online) The profile of $H_{\mathrm{cl}}^{-}(x, 0)$ (a) for $\Omega=10$ and (b) for $\Omega=10^{5}$. Different curves correspond to $g=0.1,1,2,3$ (from bottom to top). The inset in (b) zooms in the region around $x=0$.

Clearly, the Hamiltonian (1) is invariant under transformation described by $\Pi,[H, \Pi]=0$. The operator $\Pi$ has two eigenvalues \pm 1 , and corresponding eigenstates are given by

$$
\left|\varphi_{n}^{\sigma}\right\rangle \equiv \frac{\left(a^{\dagger}\right)^{n} \sigma_{x}^{n}}{\sqrt{n !}}|0, \sigma\rangle \quad(\sigma= \pm 1)
$$

Namely, $\Pi\left|\varphi_{n}^{ \pm}\right\rangle= \pm\left|\varphi_{n}^{ \pm}\right\rangle$. The Hilbert space can be decomposed into a direct sum $\mathcal{E}=\mathcal{E}_{+} \oplus \mathcal{E}_{-}$of two subspaces $\mathcal{E}_{ \pm}$ spanned by $\left|\varphi_{n}^{ \pm}\right\rangle$, respectively. Accordingly, the Hamiltonian can also be written as $H=H_{+} \oplus H_{-}$, where $H_{ \pm}$belongs to the subspace $\mathcal{E}_{ \pm}$, and the partition function $Z \equiv \operatorname{Tr}\left[e^{-\beta H}\right]$ as $Z=Z_{+}+Z_{-}$, where $Z_{ \pm} \equiv \operatorname{Tr}\left[e^{-\beta H_{ \pm}}\right]$.

Now we make a key observation, suggested in the expression for the parity eigenstates in Eq. (3), that the combination $a^{\dagger} \sigma_{x}$ behaves like a boson operator within each subspace $\mathcal{E}_{ \pm}$. To show this rigorously, we first take a dronefermion representation [21] of spin $\sigma_{z}=2 f^{\dagger} f-1$ and $\sigma_{+}=\sigma_{-}^{\dagger}=f^{\dagger}\left(d+d^{\dagger}\right)$, where $f$ and $d$ are fermion operators. We then define a new boson operator $b^{\dagger}=a^{\dagger}\left(f^{\dagger}-f\right)\left(d+d^{\dagger}\right)$ which satisfies $\left[b, b^{\dagger}\right]=1$. The parity basis states are then written as

$$
\left|\varphi_{n}^{\sigma}\right\rangle=\frac{\left(b^{\dagger}\right)^{n}}{\sqrt{n !}}|0, \sigma\rangle
$$

where $|0, \sigma\rangle$ serves as the "vacuum" of the new boson operator $b$ within $\mathcal{E}_{\sigma}$. It is now clear that the sub-block Hamiltonians $H_{ \pm}$can be rewritten solely in terms of the boson operator $b$ as

$$
H_{ \pm}=\left(b^{\dagger}-\lambda\right)(b-\lambda) \pm \frac{1}{2} \Omega \cos \left(\pi b^{\dagger} b\right) .
$$

The same expression was derived also in Ref. [20] using a canonical transformation. Having only a boson field, the expression (5) allows us to compare the model directly with corresponding classical system by deriving the classical effective potential.

To get a classical effective potential, we express the partition functions $Z_{ \pm}$in terms of a functional integration [22]. $Z_{ \pm}=\int \mathcal{D}\left[\phi^{*}(\tau), \phi(\tau)\right] \exp \left(-S_{ \pm}\right)$with the action defined by

$$
S_{ \pm}=\int_{0}^{\beta} d \tau\left[\phi^{*} \partial_{\tau} \phi+\left(\phi^{*}-\lambda\right)(\phi-\lambda) \pm \frac{1}{2} \Omega e^{-2 \phi^{*} \phi}\right]
$$

where $\beta$ is the inverse temperature, $\partial_{\tau}$ denotes the partial derivative with respect to the imaginary-time $\tau$. Making a
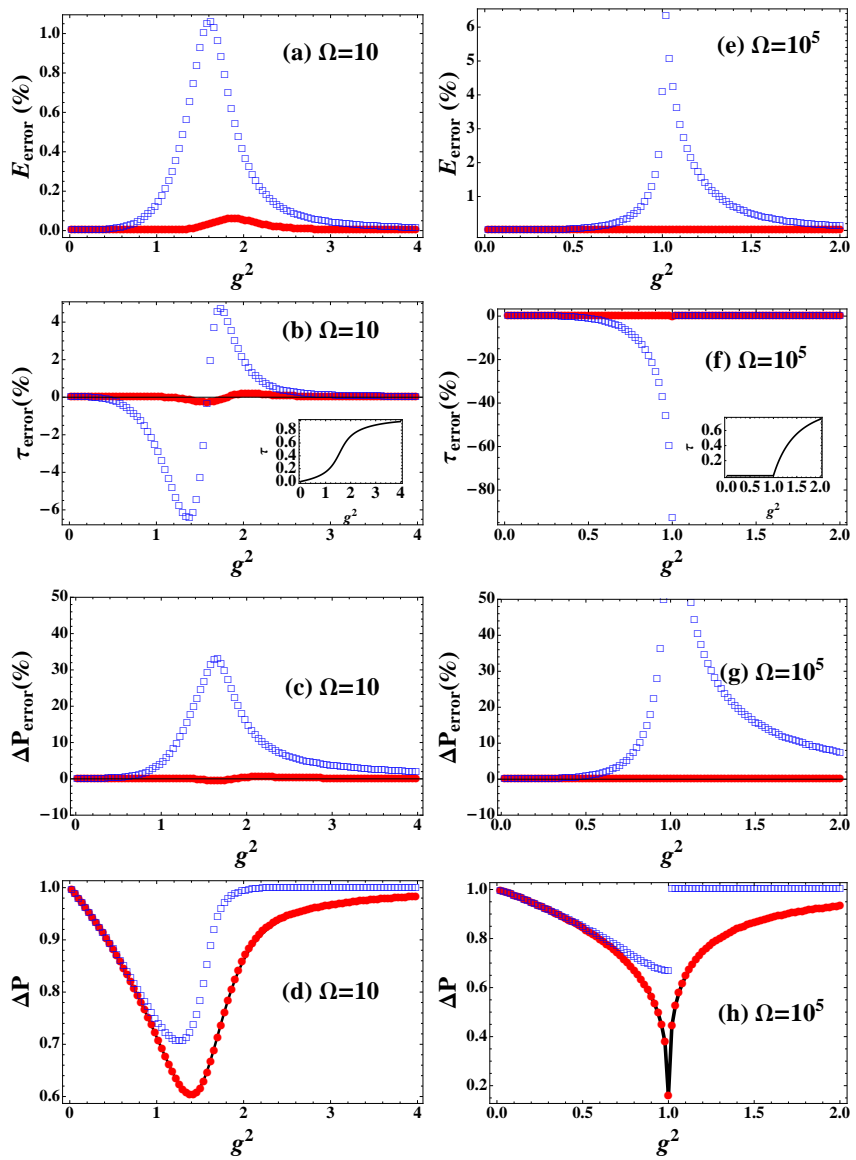

FIG. 2: (color online) Comparison of variational solutions with the exact solution. The (blue) empty square represents the DCS wave function, while the (red) filled circle indicates the DSS wave function. (a,e) Error in the energy with respect to the exact energy. (b,f) Error in the tangle. $(c, g)$ Error in the momentum variance. $(d, h)$ The momentum variance of the ground state, the (black) line indicates the exact solution.

change of variables, $p=\left(\phi^{*}+\phi\right) / \sqrt{2}$ and $p=i\left(\phi^{*}-\right.$ $\phi) / \sqrt{2}$, we can rewrite the functional integrals as $Z_{ \pm}=$ $\int \mathcal{D}[x(\tau), p(\tau)] \exp \left(-S_{ \pm}\right)$with

$$
S_{ \pm}=\int_{0}^{\beta} d \tau\left[-i p \partial_{\tau} x+H_{\mathrm{cl}}^{ \pm}(x, p)\right]
$$

Here $H_{\mathrm{cl}}^{ \pm}(x, p)$ is the effective classical Hamiltonian defined by

$$
H_{\mathrm{cl}}^{ \pm}(x, p)=\frac{1}{2}\left[(x-\sqrt{2} \lambda)^{2}+p^{2}\right] \pm \frac{1}{2} \Omega e^{-\left(x^{2}+p^{2}\right)}
$$

The potential profile is depicted in Fig. 1 for both $\Omega=10$ and $\Omega=10^{5}$. For $g \ll 1$, the classical potential has one local minimum and is highly anharmonic illustrating why a squeezed state localized around $x=0$ is a good variational solution in this regime. As $g$ approaches the critical value, 1 , it shows a bifurcation of its local minimum as in the corresponding classical system. As $g$ increases further, its local minimum around the origin disappears and a single local minimum develops at $x \sim g \sqrt{\Omega / 2}$. It is also consistent with the 
fact that the coherent state is a good approximation in this regime.

\section{VARIATIONAL SOLUTIONS}

Above we have seen from the effective classical potential that the model in Eq. (1) shows highly non-trivial behaviors in the regime $g \sim 1$. Now we investigate more closely this regime in terms of variational wave functions. To test the accuracy of a given trial wave function, we will examine not only the energy but also the quantum correlation effects such as squeezing and spin-oscillator entanglement. We characterize the squeezing effect with the momentum variance $\Delta P$ and the spin-oscillator entanglement with tangle [23] defined by $\tau=2\left[1-\operatorname{Tr}\left(\rho_{s}^{2}\right)\right]$, where $\rho_{s}$ is a reduced density matrix of the two-level system. We test the variational solution for two cases $\Omega=10$ and $\Omega=10^{5}$ by comparing it with the exact solution. The exact solution is numerically calculated by the exact diagonalization method [24], where a sufficient number of Fock basis states $|n\rangle$ (typically 300 states for $\Omega=10$ and 3000 states for $\Omega=10^{5}$ ) are kept until the desired accuracy is achieved.

In Refs. [17, 19], a superposition of two displaced coherent states was suggested as a trial wave function,

$$
\left|\psi\left(\alpha_{1}, \alpha_{2}, t\right)\right\rangle=(1-t)\left|\alpha_{1}\right\rangle+t\left|\alpha_{2}\right\rangle,
$$

where $|\alpha\rangle=D(\alpha)|0\rangle$ with $D(\alpha)=\exp \left[\alpha\left(b^{\dagger}-b\right)\right]$. Hereafter we will refer to the trial wave function in Eq. (9) as the double coherent state (DCS). It shows a reasonable accuracy for the energy (with error about 1\%) in the intermediate regime [see Figs. 2 (a) and (e)]. Although neither our effective classical potential nor a bifurcation of fixed point in the corresponding classical system can give an quantitative account for the ground state, the fact that a superposition of two wave packet is a good approximation for the ground state is consistent with the classical observations of bifurcation.

However, as shown in Figs. 2(c) and (f), the degree of entanglement between the spin and oscillator exhibits relatively larger deviations, especially for large $\Omega$.

The poor accuracy of the variational solution in Eq. (9) is even more noticeable (more than 30\%) on the momentum variance $\Delta P$ of the ground state. For both $\Omega=10$ and $10^{5}$ case, the squeezing effect is considerably underestimated in this scheme. Moreover, for the true adiabatic regime, the variational solution predicts that the squeezing effect suddenly disappears when $g$ becomes larger than 1 .

We can understand the reason for the failure by looking at the optimized variational parameters shown in Fig. 3 (e). If $g \ll 1$, the variational states are optimized at $\alpha_{1}=-\alpha_{2} \sim$ $g \sqrt{\Omega} / 2$. In this case, there is a considerable overlap between two coherent states which results in the squeezing effect. On the contrary, when $g$ becomes larger than 1, the variational parameter abruptly changes, and the value for $\alpha_{1}$ and $\alpha_{2}$ becomes an order of 10 or 100 still having opposite signs with each other. Then, the overlap between two coherent states vanishes, and cannot give the squeezing effect. This observation lead us to conclude that a deformation of individual wave packets is considerable in the true ground state, and unless the effect is taken into account in the trial wave function, the squeezing effect is inevitably underestimated.

Motivated from the above observation, we propose a superposition of two displaced-squeezed states as a trial wave function,

$$
\left|\psi\left(r_{1}, \alpha_{1}, r_{2}, \alpha_{2}, t\right)\right\rangle=(1-t)\left|r_{1}, \alpha_{1}\right\rangle+t\left|r_{2}, \alpha_{2}\right\rangle,
$$

where $|r, \alpha\rangle$ denotes the displaced-squeezed state [25] defined by $|r, \alpha\rangle=S(r)|\alpha\rangle$ with $S(r)=\exp \left[\left(r b^{\dagger} b^{\dagger}-r^{*} b b\right) / 2\right]$. We refer to the wave function in Eq. $(10)$ as the double squeezed state (DSS). Without a loss of generality, the displacement parameter $\alpha$ and the squeezing parameter $r$ are assumed to be real.

The variational parameters $r_{1}, \alpha_{1}, r_{2}, \alpha_{2}$, and $t$ are determined by minimizing the energy $E\left(r_{1}, \alpha_{1}, r_{2}, \alpha_{2}, t\right)=$ $\left\langle\psi\left|H_{-}\right| \psi\right\rangle /\langle\psi \mid \psi\rangle$. It includes two direct terms,

$$
\left\langle r_{j}, \alpha_{j}\left|H_{-}\right| r_{j}, \alpha_{j}\right\rangle=\sinh ^{2} r_{j}+\left(\lambda-\alpha_{j} e^{r_{j}}\right)^{2}-\frac{\Omega}{2} e^{-2 \alpha_{j}^{2}}
$$

for $j=1,2$, and a cross term

$$
\begin{array}{r}
\frac{\left\langle r_{1}, \alpha_{1}\left|H_{1}\right| r_{2}, \alpha_{2}\right\rangle}{\left\langle r_{1}, \alpha_{1} \mid r_{2}, \alpha_{2}\right\rangle}=\operatorname{sech}\left(r_{1}-r_{2}\right) \sinh r_{1} \sinh r_{2} \\
+\left[\lambda-\operatorname{sech}\left(r_{1}-r_{2}\right)\left(\alpha_{1} \cosh r_{2}+\alpha_{2} \sinh r_{1}\right)\right] \\
\times\left[\lambda-\operatorname{sech}\left(r_{1}-r_{2}\right)\left(\alpha_{1} \sinh r_{2}+\alpha_{2} \cosh r_{1}\right)\right] \\
-\frac{1}{2} \Omega \exp \left[-2 \operatorname{sech}\left(r_{1}-r_{2}\right) \alpha_{1} \alpha_{2}\right]
\end{array}
$$

where

$$
\begin{aligned}
& \left\langle r_{1}, \alpha_{1} \mid r_{2}, \alpha_{2}\right\rangle=\frac{\exp \left[\alpha_{1} \alpha_{2} \operatorname{sech}\left(r_{1}-r_{2}\right)\right]}{\sqrt{\cosh \left(r_{1}-r_{2}\right)}} \\
& \times \exp \left[-\frac{\left(\alpha_{1}^{2}+\alpha_{2}^{2}\right)+\left(\alpha_{1}^{2}-\alpha_{2}^{2}\right) \tanh \left(r_{1}-r_{2}\right)}{2}\right]
\end{aligned}
$$

The optimum variational parameters $r_{1}, r_{2}, \alpha_{1}, \alpha_{2}$, and $t$ thus determined are plotted as a function of $g$ in Fig. 3. The squeezing parameters $r_{1}$ and $r_{2}$ of the individual wave packets in the DSS is indeed large in the regime $g \simeq 1$, where the DCS variational solution changes abruptly. This is consistent with our prediction.

Using this variational solution, we calculate the momentum variance. The variance $\Delta P$ of the momentum $P=i\left(b^{\dagger}-b\right)$ is given by $\Delta P=\left\langle\psi\left|P^{2}\right| \psi\right\rangle /\langle\psi \mid \psi\rangle$ as in our case $\langle\psi|P| \psi\rangle=$ 0 . The direct terms and cross term are respectively given by $\left\langle r_{j}, \alpha_{j}\left|P^{2}\right| r_{j}, \alpha_{j}\right\rangle=e^{-2 r_{j}}(j=1,2)$ and

$$
\frac{\left\langle r_{1}, \alpha_{1}\left|P^{2}\right| r_{2}, \alpha_{2}\right\rangle}{\left\langle r_{1}, \alpha_{1} \mid r_{2}, \alpha_{2}\right\rangle}=\frac{2}{e^{2 r_{1}}+e^{2 r_{2}}}-\frac{4\left(\alpha_{1} e^{r_{1}}-\alpha_{2} e^{r_{2}}\right)^{2}}{\left(e^{2 r_{1}}+e^{2 r_{2}}\right)^{2}} .
$$

Thus calculated momentum variation is shown in Fig. 2 (d) and (h). The squeezing effect is accurately captured by the DSS (10), and the accuracy can be attributed to the deformation of the constituent wave packets. Our variational solution 

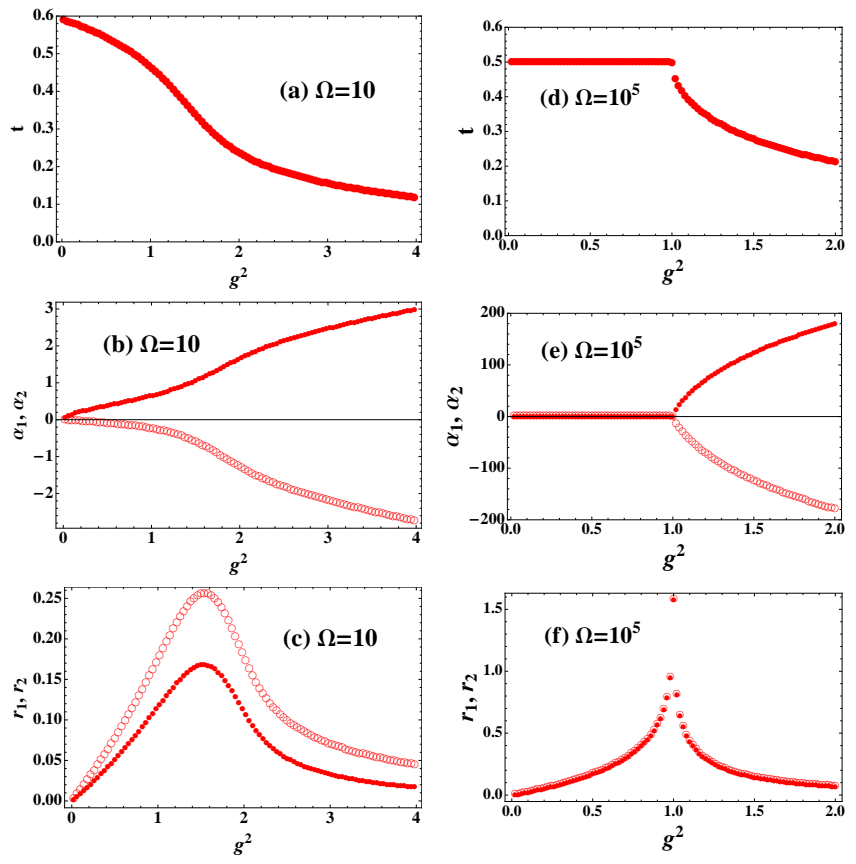

FIG. 3: (color online) Optimized variational parameters for the double squeezed state [Eq. 9] ] for $\Omega=10(\mathrm{a}, \mathrm{b}, \mathrm{c})$ and for $\Omega=10^{5}$ $(\mathrm{d}, \mathrm{e}, \mathrm{f})$. The variational parameters for the double coherent state [Eq. [10]] are qualitatively similar (not show) except for $r_{1}=r_{2}=$ 0 . (a,d) The relative weight $t$ in the superposition. (b,e) The displacement parameters $\alpha_{1}$ and $\alpha_{2}$. (c,f) The squeezing parameters $r_{1}$ and $r_{2}$.

illustrates well the phase transition-like behavior for $\Omega \rightarrow \infty$ regime.

Interestingly, the optimal variational parameters satisfy $\alpha_{1} \approx-\alpha_{2}$ and $r_{1} \approx r_{2}$ for $\Omega \gg 1$ [it is not the case for
$\Omega \sim 1$; compare Fig. $3(\mathrm{~b}, \mathrm{c})$ with Fig. $3(\mathrm{e}, \mathrm{f})]$. In this case, the DSS in Eq. (10), which has been expressed in terms of the spin-oscillator hybrid mode $b$, takes a simple form (not normalized)

$$
\begin{aligned}
|\psi(r, \alpha, r,-\alpha, t)\rangle & =(|r, \alpha\rangle+|r,-\alpha\rangle) \otimes|\downarrow\rangle \\
+ & (1-2 t)(|r, \alpha\rangle-|r,-\alpha\rangle) \otimes|\uparrow\rangle
\end{aligned}
$$

in terms of the original spin and oscillator mode. This form shows transparently the entanglement feature in the optimal wave function: For $g \ll 1, t \approx 1 / 2$ and the spin and oscillator is separable. As $g$ increases, $t$ decreases, which leads to strong entanglement between spin and oscillator mode as shown in the insets of Fig. 2 (c) and (g). and the overlap between $|\alpha\rangle$ and $|-\alpha\rangle(\alpha \sim \lambda)$ gives a finite amount of squeezing.

\section{DISCUSSION}

We have studied the spin-boson model (1) in the ultrastrong coupling limit by means of an effective classical potential and an improved variational wave function.

We note that in the true adiabatic regime $(\Omega \gg 1)$, the critical strength $g$ for the entanglement coincide with the point where the squeezing effect is maximum. This coincidence can also be noticed from the crossover behavior of the effective classical potential (9). Nevertheless, the precise relation among the bifurcation in the classical potential in Fig. (1) or in the corresponding classical model [12], the squeezing effect, and the entanglement remains an interesting open problem in the ultra-strong coupling limit of the model (1).

This work has been supported by the NRF Grant (No. 20090080453), the BK21 Program, and the APCTP.
[1] J. M. Raimond, M. Brune, and S. Haroche, Rev. Mod. Phys. 73, 565 (2001).

[2] M. D. LaHaye, J. Suh, P. M. Echternach, K. C. Schwab, and M. L. Roukes, Nature 459, 960 (2009).

[3] E. T. Jaynes and F. W. Cummings, Proc. IEEE 51, 89 (1963).

[4] H. P. Yuen, Phys. Rev. A 13, 2226 (1976).

[5] A. Zazunov, D. Feinberg, and T. Martin, Phys. Rev. Lett. 97, 196801 (2006).

[6] M.-J. Hwang, J.-H. Choi, and M.-S. Choi, 1004.1497 (2010).

[7] S. Ashhab and F. Nori, Phys. Rev. A 81, 042311 (2010).

[8] M. Devoret, S. Girvin, and R. Schoelkopf, Annalen der Physik 16, 767 (2007).

[9] J. Bourassa, J. M. Gambetta, A. A. Abdumalikov, O. Astafiev, Y. Nakamura, and A. Blais, Phys. Rev. A 80, 032109 (2009).

[10] D. Zueco, G. M. Reuther, S. Kohler, and P. Hänggi, Phys. Rev. A 80, 033846 (2009).

[11] G. Levine and V. N. Muthukumar, Phys. Rev. B 69, 113203 (2004).

[12] A. P. Hines, C. M. Dawson, R. H. McKenzie, and G. J. Milburn, Phys. Rev. A 70, 022303 (2004).

[13] G. Liberti, R. L. Zaffino, F. Piperno, and F. Plastina, Phys. Rev. A 73, 032346 (2006).
[14] C. P. Meaney, T. Duty, R. H. McKenzie, and G. J. Milburn, Phys. Rev. A 81, 043805 (2010).

[15] E. K. Irish, Physical Review Letters 99, 173601 (pages 4) (2007).

[16] F. Pan, X. Guan, Y. Wang, and J. P. Draayer, A progressive diagonalization scheme for the rabi hamiltonian, arXiv:1004.2801 (2010).

[17] H. Chen, Y.-M. Zhang, and X. Wu, Phys. Rev. B 40, 11326 (1989).

[18] H. B. Shore and L. M. Sander, Phys. Rev. B 7, 4537 (1973).

[19] J. Stolze and L. Müller, Phys. Rev. B 42, 6704 (1990).

[20] G. Benivegna and A. Messina, Phys. Rev. A 35, 3313 (1987).

[21] H. J. Spencer and S. Doniach, Phys. Rev. Lett. 18, 994 (1967).

[22] J. W. Negele and H. Orland, Quantum Many-Particle Systems (Addison-Wesley, Redwood, 1988).

[23] V. Coffman, J. Kundu, and W. K. Wootters, Phys. Rev. A 61, 052306 (2000).

[24] One may also use recently proposed alternative methods in Refs. [15, 16].

[25] M. O. Scully and M. S. Zubairy, Quantum Optics (Cambridge University Press, Cambridge, 1997). 